\title{
Pyopneumopericardium as a complication of oesophagogastrostomy
}

\author{
OTTO GAGO, PARMAJEET S. CHOPRA, LEE H. ELLISON, \\ and PHILIP J. SILVERMAN
}

Department of Surgery, Section of Thoracic Surgery, and Department of Radiology, University Hospital, University of Michigan Medical Center, Ann Arbor, Michigan 48104, USA

A case of pyopneumopericardium 13 years following resection of a peptic stricture and oesophagogastrostomy, and treated successfully by closure of the gastric ulcer, pericardiectomy, and drainage, is reported.

Pyopneumopericardium is a rare entity and is even more infrequent as a complication of benign gastric ulceration following oesophagogastrostomy.

The usual course of events is the perforation of a gastric ulcer with subphrenic abscess formation and dissection through the diaphragm into the pericardial sac. The cases reported in the literature are associated with extension of carcinoma of the lung, oesophagus, and stomach, trauma, iatrogenic causes, perforation of inflammatory lesions within the chest as a complication of hyaline membrane disease in the newborn, and in newborn infants assisted by artificial ventilation (Cramm and Robinson, 1971 ; Dickson and Girling-Butcher, 1960 ; Dons, Eriksen, Ryssing, and Therkelsen, 1964 ; Mathieu, Nussle, Torrado, and Sadeghi, 1970; Romhilt and Alexander, 1965 ; Specter, Danesh, and Sterling, 1968).

Shackelford reviewed 77 cases in great detail in 1931 and classified the condition according to aetiology. In 1968 Wegryn, Zaroff, and Weiner reported a patient with pyopneumopericardium who had had a transthoracic repair of hiatus hernia for oesophagitis and gastrointestinal bleeding six years earlier and developed an ulcer in the fundus of the stomach with subsequent perforation into the pericardial sac. The patient was treated with initial pericardiotomy and drainage followed by oesophagogastrostomy. As far as we know, this was the first patient who survived surgical correction of this complication.

We believe that our case is unique because not only is the pyopneumopericardium a complication of oesophagogastrostomy for benign oesophageal stricture but the patient survived definitive surgical correction.

\section{CASE REPORT}

B.F., a 52-year-old Mexican man, was admitted to thew University of Michigan Medical Center in August 1957 with a three-week complaint of dysphagia foro solid food. Radiographic examination of the uppers gastrointestinal tract showed a stricture at the cardio-oesophageal junction. The patient underwento oesophagoscopy and biopsy on two occasions. Histo $\mathbb{2}$ logical examination of the biopsy material revealed peptic oesophagitis. On 26 August 1957 an oesophago $\frac{3}{3}$ gastrostomy was performed through a left thora cotomy. No neoplasm was found in the surgica specimen.

Six years later he was again admitted to hospita when he developed dysphagia that was progressive to the point of his being able to take only liquids. He had a history of progressive weight loss in the yea before this admission. An upper gastrointestinal series?. demonstrated a stricture that appeared benign at the level of the oesophagogastric anastomosis. When oesophagoscopy was performed an apparently benigy stricture was seen $35 \mathrm{~cm}$ from the incisors. Treatmen consisted of a bland diet regimen and progressive dilatations.

The patient was seen four-and-one-half years lateñ when he again presented with a one-year history of progressive dysphagia. At that time he was admitted to another hospital with complete oesophageal obstruc స્心 tion, and a feeding gastrostomy was done. He was transferred to the University of Michigan HospitaF where oesophagoscopy showed a recurrent stricture An exploratory thoracotomy was performed in Januar 1969, revealing total oesophageal obstruction secondary to inflammatory fibrosis. He underwent pyloro 0 plasty and revision of his oesophagogastrostomy. The patient's postoperative course was uncomplicated an on discharge from hospital he was able to swallow food of any kind.

On 11 March 1970, he was admitted to the generar 


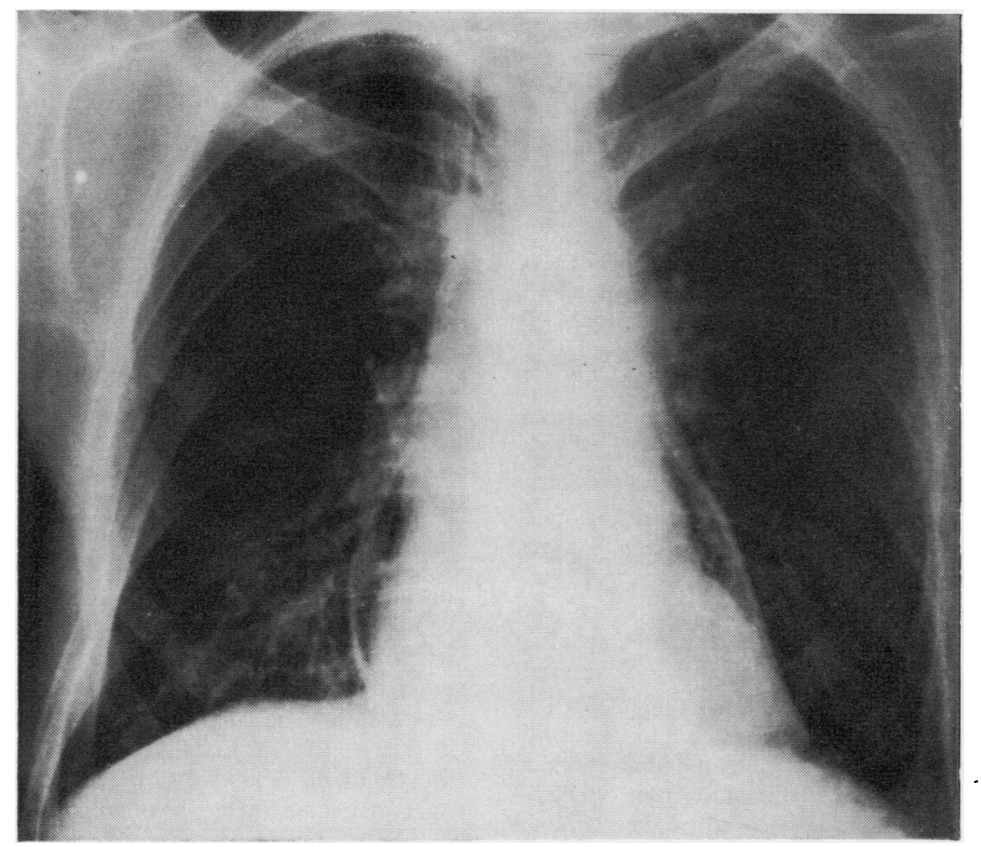

FIG. 1. Chest radiograph on admission showing air in the pericardial sac.

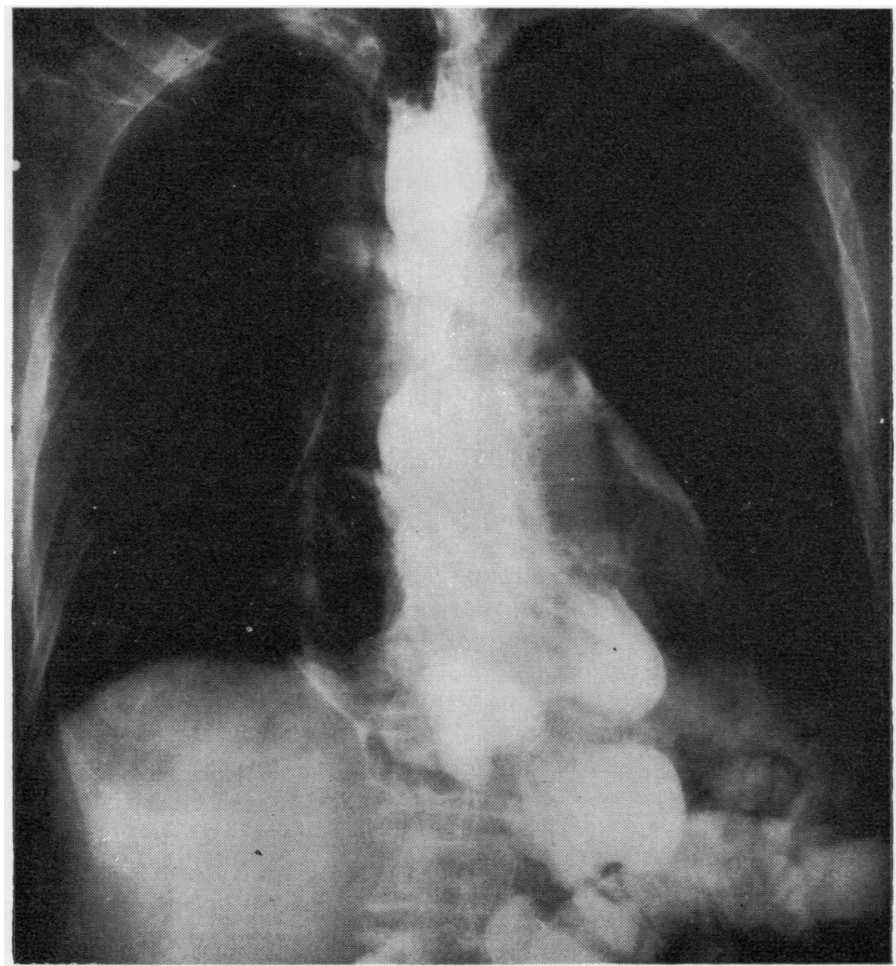

FIG. 2. Upper gastrointestinal tract examination showing contrast material in the pericardial sac and a large gastric ulcer in the supradiaphragmatic portion of the stomach. 
surgical service for repair of a left inguinal hernia. He gave a three months' history of intermittent epigastric pain radiating to both shoulders. A thorough evaluation of the oesophagogastric anastomosis failed to reveal any obvious abnormality.

Two months after discharge the patient presented with a two-hour history of a pressure-like substernal chest pain radiating into both arms associated with dyspnoea and diaphoresis. This episode subsided spontaneously only to recur the next morning in association with vomiting. The pain was aggravated by lying down and improved only minimally with antacids. There was no previous history of coronary artery disease.

On admission he appeared acutely and chronically ill. The vital signs were: blood pressure 100/60 $\mathrm{mmHg}$; pulse 92 beats/minute, regular; respiratory rate $20 /$ minute; and temperature $100 \cdot 6^{\circ} \mathrm{F}\left(38 \cdot 1^{\circ} \mathrm{C}\right)$. Physical examination revealed: increased anterior posterior diameter of the chest; a well-healed left thoracotomy scar; the point of maximum cardiac impulse in the fifth left intercostal space in the midclavicular line; decreased breath sounds and râles at the left base and a scratching sound coincident with the second heart sound. An electrocardiogram showed ST and $T$ wave changes consistent with pericarditis. The chest radiograph on admission disclosed air in the pericardial sac (Fig. 1). Radiographic findings following ingestion of $150 \mathrm{ml}$ of $30 \%$ Hypaque showed narrowing of the oesophagogastric junction, a $5 \mathrm{~cm}$ accumulation of contrast material along the anterior wall of the stomach adjacent to the inferior aspect of the pericardium, and contrast material outlining the heart and the pericardial cavity (Fig. 2). A diagnosis was made of stenosis of the oesophagogastric junction and a large gastric ulcer with erosion into the pericardial cavity.

The patient's blood pressure dropped to $66 / 42$ $\mathrm{mmHg}$ and on the day of admission an exploratory left thoracotomy was performed. During induction of anaesthesia the blood pressure dropped to $40 \mathrm{mmHg}$ systolic and there was no effective heart beat. He responded to external cardiac massage, endotracheal intubation, and vasopressors. The chest was opened through the old seventh interspace incision. Pleural adhesions were broken down. The stomach was identified in a retrocardiac position and the line of anastomosis was intact. There was marked inflammation of the mediastinal structures and purulent drainage from behind the heart. Because of difficulty in locating the area of perforation a gastrostomy was done. An ulcer, $3.0 \mathrm{~cm}$ in diameter, was found in the supradiaphragmatic portion of the stomach. There was a $1.0 \mathrm{~cm}$ perforation at the posterior wall of the pericardial sac. The sac contained a large amount of pus. After irrigating the pericardial sac, the gastric perforation was biopsied and closed from within the pericardium and reinforced with sutures through the gastric lumen. The suture line was protected with pericardial fat. The chest was drained with apical and basilar chest tubes and one retrocardiac pericardial tube.
Histological examination of the biopsy material revealed a benign gastric ulcer. Cultures of tissue obtained at operation grew Streptococcus viridans, coagulase-negative staphylococcus, Streptococcus lactis, and klebsiella. The pericardial drainage was 715 $\mathrm{ml}$ on the first postoperative day but decreased quickly thereafter. By the eighth postoperative day the patient had stabilized, the apical chest tube was out but the pericardial tube was still draining purulent material. On the thirteenth postoperative day the patient's condition deteriorated rapidly. His blood pressure dropped to $74 / 60 \mathrm{mmHg}$ with a $40 \mathrm{mmHg}$ fluctuation of systolic pressure between inspiration and expiration (pulsus paradoxus); the neck veins were distended at $30^{\circ}$; his level of consciousness decreased; his skin was cool and clammy and he developed a loud pericardial rub.

On the same day a diagnosis of constrictive pericarditis was made. He was reexplored through the seventh intercostal space wound. The previously placed pericardial tube was removed, revealing a large amount of pus in the pericardial cavity. The pericardium was thickened to 5 to $10 \mathrm{~mm}$ in several places and was markedly restricting the action of the left ventricle. A pericardiectomy was performed with immediate improvement of heart function. The previous closure of the gastric perforation was intact. Apical and basilar chest tubes were inserted in addition to two dependent pericardial tubes. A Foley catheter was left in the area at the base of the heart for the purpose of irrigation with antibiotics. Cultures at the time of the operation grew coagulase-positive staphylococcus and Streptococcus faecalis. Pericardial irrigations were maintained for two weeks after the operation. The patient continually improved.

By the twenty-sixth postoperative day there was no further drainage and the chest tubes were removed. Ten days later a sinus tract injection through a pericardial tube revealed a large cavity surrounding the heart. One pericardial drain was left. He was discharged on 30 June 1970, 56 days after admission.

The patient has had a satisfactory postoperative course under conservative management of the gastric ulcer and stenosis of the oesophagogastromy.

\section{DISCUSSION}

A retrospective view of the clinical history reveals o that the cause of anterior chest and epigastric $N$ pain symptoms in this patient were related to a N large gastric ulcer in the intrathoracic segment of $\sigma$ the stomach that was not recognized in the upper gastrointestinal examination in March 1970. When the patient returned in May 1970 with severe chest pain, hypotension, signs of acute pericarditis, and the chest film showing a pneumopericardium, it was obvious that a possible gastric perforation into the pericardial cavity had occurred, as was shown by the upper gastrointestinal examination at this later time. On sur- 
gical exploration we found a severe fibrinopurulent pericarditis with a $1 \times 1 \mathrm{~cm}$ perforation at the posterior wall of the pericardial sac communicating with a $3 \times 3 \mathrm{~cm}$ ulcer of the anterior wall of the intrathoracic stomach. The initial treatment of pericardial drainage and suture of the ulcer was a life-saving procedure, but it had to be followed by pericardiectomy two weeks later when the patient developed acute tamponade in spite of adequate drainage, at which time an upper tube and two lower tubes were left in the pericardial space for postoperative irrigation with antibiotics. This last measure has proved its value in the treatment of severe purulent pericarditis in children at our institution.

We wish to point out this possible complication of oesophagogastrostomy, especially in those patients who have had this operation for benign disease and in whom prolonged survival is expected. Should this complication occur, the treatment must be immediate surgical exploration with aggressive drainage of the pericardial space. The massive insult of the gastric content in the pericardial sac in our case produced a violent chemical and inflammatory reaction of the pericardium leading to a restrictive pericarditis two weeks after closure of the ulcer and drainage of the pericardium. The operative findings of a thick fibrotic pericardium make us believe that pericardiectomy should be included as a part of the initial therapy as the technical difficulties encountered at the time of the initial insult are minimal.

\section{REFERENCES}

Cramm, R. E., and Robinson, F. W. (1971). Pneumopericardium associated with gastric ulcer. Report of a case and literature review. Gastroenterology, 60, 311.

Dickson, D. S. P., and Girling-Butcher, M. (1960). Spontaneous pneumopericardium. N.Z. med.J., 59, 250.

Dons, N., Eriksen, N. R., Ryssing, E., and Therkelsen, F. (1964). Pneumopericardium with oesophagopericardial fistula. Report of a case with recovery. Acta chir. scand., 128, 766.

Mathieu, J. M., Nussle, D., Torrado, A., and Sadeghi, H. (1970). Pneumopericardium in the newborn. Pediatrics, 46, 117.

Romhilt, D. W., and Alexander, J. W. (1965). Pneumopericardium secondary to perforation of benign gastric ulcer. Amer. med. Ass., 191, 140.

Shackelford, R. T. (1931). Hydropneumopericardium: report of a case with a summary of the literature. J. Amer. med. Ass., 96, 187.

Specter, J., Danesh, S., and Sterling, J. A. (1968). Pneumopericardium due to abdominal trauma. Int. J. Surg., 50, 145.

Wegryn, R. L., Zaroff, L. I., and Weiner, R. S. (1968). Spontaneous tension pneumopericardium. New Engl. J. Med., 279, 1440. 